\title{
Hidratación oral con una solución a base de harina de plátano precocida con electrolitos estandarizados
}

\author{
Carlos Bernal ${ }^{1}$, Gloria Margarita Alcaraz ${ }^{2}$, Jorge Eliécer Botero ${ }^{1}$ \\ ${ }^{1}$ Departamento de Pediatría y Puericultura, Universidad de Antioquia, Medellín, Colombia. \\ ${ }^{2}$ Facultad de Enfermería, Universidad de Antioquia, Medellín, Colombia. \\ El trabajo se realizó en la Unidad Vida Infantil del Hospital Francisco Valderrama de Turbo, Antioquia.
}

Objetivo. El objetivo del presente trabajo fue determinar si una solución de hidratación oral a base de harina de plátano precocida con electrolitos, además de ser efectiva para tratar la deshidratación, tiene propiedades antidiarreicas.

Material y métodos. Se incluyeron 101 niños con edades entre 1 y 41 meses con diarrea de menos de una semana y deshidratación leve, y sin complicaciones asociadas. El grupo 1 (54 niños) recibió sales de rehidratación oral de la Organización Mundial de la Salud (SRO/OMS); el grupo 2 (47 niños) recibió solución con concentraciones de electrolitos similares pero que, en lugar de la glucosa, contiene $50 \mathrm{~g}$ de harina de plátano precocida (sales de rehidratación oral/plátano (SRO/plátano).

Resultados. Se hidrató con éxito el $94,4 \%$ de los niños en el grupo SRO/OMS, y el $91,5 \%$ $(p=0,70)$ del grupo SRO/plátano. El tiempo necesario para lograr la hidratación fue de 5,8 horas $(D E= \pm 3,0)$ en el grupo SRO/OMS y de 6,2 horas $(D E: \pm 3,7)$ en el grupo SRO/plátano $(p=0,31)$. El gasto fecal hasta alcanzar la hidratación en el grupo SRO/OMS fue de $7,5 \mathrm{~g} / \mathrm{kg}$ por hora $(\mathrm{DE}= \pm 7,6)$, y en el grupo SRO/plátano de $7,05 \mathrm{~g} / \mathrm{kg}$ por hora $(\mathrm{DE}= \pm 9,4)(p=0,78)$. No hubo diferencia significativa en el sodio plasmático.

El período de observación para el grupo SRO/OMS fue de 22,3 horas (DE= $\pm 5,3$ ), y de 22,7 horas $(\mathrm{DE}= \pm 4,7)$ para el grupo $\mathrm{SRO} /$ plátano. El gasto fecal durante el período de observación fue de $5,7 \mathrm{~g} / \mathrm{kg}$ por hora $(\mathrm{DE}= \pm 4,7)$ para el grupo SRO/OMS y de $6,3 \mathrm{~g} / \mathrm{kg}$ por hora $(\mathrm{DE}= \pm 7,9)$ para el grupo $S R O / p l a ́ t a n o ~(p=0,67)$. La proporción de niños que necesitaron líquidos endovenosos durante las primeras veinticuatro horas fue de $11,1 \%$ en el grupo SRO/OMS y de $8,5 \%$ en el grupo SRO/plátano ( $R R=1,31$; IC 95\%: 0,39 a 4,35).

Conclusiones. La solución a base de harina de plátano es efectiva y segura para corregir la deshidratación. No se pudo demostrar en este estudio el efecto antidiarreico de la solución a base de harina de plátano.

Palabras clave: deshidratación, diarrea infantil, soluciones para rehidratación, fluidoterapia.

Oral rehydration with a plantain flour-based solution precooked with standardized electrolytes

Background. The success of oral rehydration therapy in cases of severe diarrheas have led to a need for locally based therapy preparations. A preparation consisting of a precooked plantain flour-based solution and standard rehydration electrolytes was examined for its effectiveness in treating dehydration, as well as its anti-diarrhoeal properties.

Methods. A sample of 101 children, ranging in age from 1 to 48 months, was selected, which had presented diarrhea for less than one week from onset, with mild dehydration and no associated complications. Fifty-four children received the ORS/WHO standard treatment, and 47 received a solution with electrolytes similar to ORS $/ \mathrm{WHO}$, containing $50 \mathrm{~g}$ of precooked plantain flour instead of glucose (ORS/plantain).

Results. Successful rehydration was achieved in $94.4 \%$ of the children in the ORS/WHO group and $91.5 \%$ in the ORS/plantain group $(p=0.70)$. Required time for rehydration was 5.8 (SD $\pm 3.0)$ hours in the ORS/WHO group and $6.2(\mathrm{SD} \pm 3.7)$ hours in the ORS/plantain group $(p=0.31)$. Stool output during rehydration was $7.5 \mathrm{~g} / \mathrm{kg}$ body weight/hour (SD \pm 7.6$)$ in the ORS/WHO 
group, and $7.05 \mathrm{~g} / \mathrm{kg} / \mathrm{hour}(\mathrm{SD} \pm 9.4)$ in the ORS/plantain group $(\mathrm{p}=0.78)$. No significant differences were noted in the concentrations of plasma sodium. The observation period was 22.3 hours $(\mathrm{SD} \pm 5.3)$ for the ORS/WHO group and 22.7 hours $(\mathrm{SD} \pm 4.7)$ for the ORS/plantain group. Stool output during the observation period was $5.7 \mathrm{~g} / \mathrm{kg} / \mathrm{hour}(\mathrm{SD} \pm 4.7)$ in the ORS/WHO group and $6.3 \mathrm{~g} / \mathrm{kg} / \mathrm{hour}(\mathrm{SD} \pm 7.9)$ in the ORS/plantain group $(\mathrm{p}=0.67)$. The proportion of children requiring intravenous fluids during the first 24 hours was $11.1 \%$ in the ORS/WHO group and $8.5 \%$ in the ORS/plantain group ( $\mathrm{RR}=1.31 ; 95 \% \mathrm{Cl} 0.39-4.35)$.

Conclusions. The plantain flour-based solution proved effective and safe in correcting dehydration; however the antidiarrhoeal properties of ORS/plaintain were not demonstrated.

Key words: dehydration, infantile diarrhea, rehydration solutions, fluid therapy, plaintain flour.

Las enfermedades diarreicas continúan apareciendo entre las primeras causas de mortalidad y morbilidad en los niños menores de cinco años en los países en vía de desarrollo (1). En el departamento de Antioquia, Colombia, la tasa de mortalidad por diarreas en 1997 en menores de cinco años fue de 19,1 por 100.000, mientras que en uno de sus municipios, Chigorodó, fue de 420,56 por 100.000 (2).

A pesar de sus ventajas, el suero de rehidratación oral (SRO) con la fórmula recomendada desde 1975 por la Organización Mundial de la Salud (OMS) no disminuye ni la cantidad ni la duración de la diarrea. Desde entonces se ha venido buscando un suero que, además de hidratar y prevenir la deshidratación, disminuya la diarrea en cantidad y duración y que, preferiblemente, tenga algún efecto positivo sobre la nutrición. Se han hecho muchos estudios en busca de una solución de hidratación oral mejorada; se han ensayado sueros en los cuales la glucosa ha sido reemplazada por aminoácidos, dipéptidos, tripéptidos, hidrolizados de proteínas, arroz, plátano y otros alimentos. También se han estudiado sueros con menores concentraciones de glucosa y sodio, buscando que tengan una menor osmolaridad (3-14).

Se ha establecido que un suero que, en lugar de contener $20 \mathrm{~g}$ de glucosa, tenga entre $50 \mathrm{~g} \mathrm{y} 80 \mathrm{~g}$ de polvo de arroz y concentraciones de electrolitos

Correspondencia:

Carlos Bernal, Departamento de Pediatría y Puericultura, Universidad de Antioquia, Apartado aéreo 1226, Medellín, Colombia

Telefax: (574) 2637885.

cabp@epm.net.co

Recibido: 31/05/04; aceptado: 11/10/04 similares a los del suero oral, disminuye el volumen y la duración de la diarrea, pero solamente en pacientes con cólera; en diarrea no colérica no presenta ventajas sobre la solución estándar (4).

Un estudio bioquímico de una solución de rehidratación oral preparada con harina de plátano, llevado a cabo con cuatro variedades de plátano verde: hartón (Musa paradisiaca), dominico (Musa negra), guineo (Musa coccinea Andrade) y primitivo (Musa sp.), mostró que las concentraciones de glucosa por digestión de los polisacáridos, sodio y potasio eran similares en todas las variedades de plátano. Por ello, se estandarizó la preparación SRO con $50 \mathrm{~g}$ de harina de plátano de cada de variedad y $3,5 \mathrm{~g}$ de sal en $1.100 \mathrm{ml}$ de agua y 12 minutos de ebullición; las osmolaridades de las soluciones preparadas en esta forma casera fueron igualmente similares (15).

A partir de esta investigación, nuestro grupo adelantó en 1995 un estudio con el objetivo de comparar la eficacia de una solución a base de harina de plátano con la fórmula casera estandarizada, en relación con la solución de hidratación oral recomendada por la OMS (9). El hallazgo más llamativo de ese estudio fue que el gasto fecal fue significativamente menor entre los niños que recibieron la solución a base de harina de plátano (9). Sin embargo, el tiempo de observación fue relativamente corto, ya que se registró solamente durante el período de hidratación. Para poder confirmar el efecto antidiarreico de la solución a base de harina de plátano, cuya osmolaridad era de $134,4 \mathrm{mOsm} / \mathrm{L}$ $(\mathrm{DE}=12,3)$, las mediciones deberían hacerse, por lo menos, durante las primeras 24 horas.

En relación con los sueros de baja osmolaridad, se ha encontrado que disminuyen la necesidad 
de utilizar líquidos intravenosos durante el episodio de diarrea y disminuyen el gasto fecal (16); por tal motivo, el comité de expertos de la OMS/Unicef recomendó el cambio de la formulación por una solución con $245 \mathrm{mOsm} / \mathrm{L}$, en la cual el sodio aporta $75 \mathrm{mmol} / \mathrm{L}$ y la glucosa $75 \mathrm{mmol} / \mathrm{L}$ (17).

Para el presente estudio se utilizó un suero preparado con $50 \mathrm{~g}$ de harina de plátano precocido con adición de una mezcla de electrolitos con la misma composición del suero oral estándar, con el objetivo determinar si la solución de hidratación oral a base de harina de plátano precocida con electrolitos, además de ser efectiva para tratar la deshidratación producida por las enfermedades diarreicas en los niños, tiene propiedades antidiarreicas.

\section{Materiales y métodos}

Se llevó a cabo un estudio clínico controlado. Se incluyeron 101 niños con edades comprendidas entre 1 y 48 meses que presentaban diarrea de menos de siete días de duración, con deshidratación y sin choque hipovolémico.

El estudio se adelantó en la Unidad Vida Infantil que es un programa de la Universidad de Antioquia y del Hospital Francisco Valderrama del municipio de Turbo (Antioquia), entre marzo de 2001 y julio de 2002. El protocolo fue aprobado por el comité de ética del Centro de Investigaciones Médicas de la Universidad de Antioquia.

La variable utilizada para calcular el tamaño de la muestra fue el gasto fecal durante las primeras veinticuatro horas, con base en los resultados del estudio de Guiraldes, realizado en Chile, citado en el metaanálisis de los sueros a base de arroz (4), en pacientes similares a los incluidos en este estudio en quienes el gasto fecal durante las primeras 24 horas en niños que recibieron la solución estándar fue de $126 \mathrm{~g} / \mathrm{kg}(\mathrm{DE}= \pm 64)$. Se consideró deseable una disminución del $30 \%$ en el gasto fecal entre los niños que recibían la solución experimental en relación con la estándar. Para calcular el tamaño de la muestra se utilizó la fórmula recomendada por Pocock (18), y se aceptó un error de tipo I del 0,05, y un error de tipo Il de 0,20; por consiguiente, se necesitarían 45 niños por cada grupo.
El estado de hidratación se evaluó de acuerdo con los parámetros establecidos por la Estrategia de Atención Integrada a las Enfermedades Prevalentes de la Infancia (Aiepi) de la OMS (19). Los criterios de inclusión fueron los siguientes:

niños con edades entre 1 y 48 meses; no se incluyeron niñas por la dificultad en la medición precisa de la eliminación urinaria;

diarrea con una duración menor de siete días, con deshidratación y sin choque hipovolémico;

historia de haber presentado, por lo menos, tres deposiciones líquidas o semilíquidas en las veinticuatro horas anteriores al ingreso al hospital;

ausencia de otras enfermedades graves asociadas; $y$

aceptación de la participación en el estudio por parte de la familia.

Se excluyeron los niños que presentaban los siguientes criterios:

niños con desnutrición edematosa (kwashiorkor);

niños que hubieran participado antes en el estudio y que volvieron deshidratados;

niños que presentaran al ingreso cuadro clínico sospechoso de íleo paralítico.

Una vez verificados los criterios de inclusión y obtenido el consentimiento informado por parte de los padres del niño, se procedió a la asignación aleatoria. Personas diferentes a los investigadores prepararon una lista maestra de asignación al azar utilizando bloques permutados de longitud variable, entre 8 y 12 por grupo. Con base en la lista maestra se prepararon sobres cerrados de papel opaco, numerados en forma consecutiva. En los sobres se incluyó el nombre del suero que debería recibir el niño.

Una vez realizada la asignación aleatoria, los niños se pesaron y se midieron y se inició la hidratación.

La solución para el grupo experimental se preparó con plátano hartón ( $M$. paradisiaca) precocido en agua y luego secado y pulverizado; la solución (SRO/plátano) se preparó así: $1.100 \mathrm{ml}$ de agua, 
$50 \mathrm{~g}$ de harina de plátano precocida estabilizada en agua con goma xantán al $0,3 \%$, con adición de $90 \mathrm{mmol} / \mathrm{L}$ de sodio, $80 \mathrm{mmol} / \mathrm{L}$ de cloruro, 20 $\mathrm{mmol} / \mathrm{L}$ de potasio y $10 \mathrm{mmol} / \mathrm{L}$ de citrato, pero sin glucosa. La osmolaridad de la solución preparada fue de $226 \mathrm{mOsm} / \mathrm{L}(\mathrm{DE}= \pm 9,16)$. El grupo control recibió el suero de rehidratación oral de la OMS (SRO/OMS), que contiene $90 \mathrm{mmol} / \mathrm{L}$ de sodio, $80 \mathrm{mmol} / \mathrm{L}$ de cloruro, $20 \mathrm{mmol} / \mathrm{L}$ de potasio, $10 \mathrm{mmol} / \mathrm{L}$ de citrato y $111 \mathrm{mmol} / \mathrm{L}$ de glucosa, con osmolaridad de $311 \mathrm{mOsm} / \mathrm{L}$.

A los niños se les ofreció entre 50 y $100 \mathrm{ml} / \mathrm{kg}$ de suero durante las primeras cuatro a seis horas, y la cantidad fue mayor o menor según libre demanda, utilizando el volumen necesario hasta conseguir la hidratación. La madre administró las soluciones con vaso y cuchara en forma continua, bajo la supervisión y con el apoyo del personal médico y de enfermería.

En los primeros minutos después de iniciada la hidratación, se tomó una muestra de sangre para determinar los valores de sodio y potasio. También se tomaron muestras de materia fecal para practicar el examen coprológico, coprocultivo y determinar presencia de rotavirus.

Los niños fueron evaluados cada hora durante las primeras ocho horas y, luego, cada dos horas hasta completar las primeras veinticuatro horas después de la hospitalización. El peso se registró al ingreso, cuando se consideró que el niño estaba hidratado y al final del período de observación. $\mathrm{Al}$ terminar la hidratación se tomaron de nuevo muestras de sangre para medir el sodio y el potasio.

Una vez hidratados los niños, se continuó la reposición de las pérdidas concomitantes con la misma solución utilizada para la rehidratación y se inició la alimentación con una dieta estandarizada de acuerdo con la edad.

El tratamiento de los pacientes después de las primeras veinticuatro horas se continuó, según la evolución, en forma ambulatoria o en el hospital.

La clasificación del riesgo nutricional se realizó después de conseguida la hidratación de acuerdo con las normas del National Center Health Statistics (NCHS) (20). Se utilizó el indicador de desnutrición aguda peso para la talla $(p / t)$. El análisis se hizo mediante el puntaje $Z$ con base en el programa Epinut. El punto de corte de este indicador fue de menos una desviación estándar.

Se consideró un éxito cuando la hidratación se consiguió por vía oral o por sonda en un tiempo máximo de 12 horas.

Los niños que no pudieron hidratarse por vía oral ni por sonda nasogástrica recibieron hidratación parenteral; estos casos se consideraron fracasos tempranos.

Se consideró como fracaso tardío cuando los niños volvieron a deshidratarse y necesitaron líquidos parenterales durante el período de observación.

La medición del gasto fecal se hizo mediante el empleo de pañales desechables prepesados. La recolección de orina se hizo con bolsas recolectoras. La medición del volumen de los vómitos se hizo mediante el empleo de paños prepesados.

La longitud de los niños se determinó por infantómetro con sensibilidad de $1 \mathrm{~mm}$. El peso se determinó en balanza mecánica Health o Meter con sensibilidad de $5 \mathrm{~g}$.

El peso de los pañales y de los paños utilizados para secar los vómitos se hizo en una balanza Ohaus Triple Beam Balance, calibrada de gramo en gramo hasta $500 \mathrm{~g}$.

El almacenamiento de los datos se hizo utilizando el programa Epiinfo, versión 6.0.1 (CDC, Atlanta, USA).

\section{Exámenes de laboratorio}

Las muestras para las mediciones de sodio y potasio plasmático se centrifugaron y se congeló el suero. Se hicieron envíos periódicos de las muestras congeladas al Laboratorio de Nefrología Pediátrica del pabellón infantil del Hospital Universitario San Vicente de Paúl de Medellín, donde se hicieron la mediciones en fotómetro de llama Corning $\circledR_{\text {. }}$.

Las muestras de materia fecal se obtuvieron por estímulo rectal y se enviaron al laboratorio clínico del Hospital Francisco Valderrama de Turbo; la 
presencia de parásitos se determinó mediante examen en fresco con solución salina al 0,9\% y lugol. La búsqueda de rotavirus se efectuó empleando el estuche The Murex Rotavirus Latex Test $\mathbb{R}^{-}$

Se hizo coloración de Gram modificado para Campylobacterspp. y de Zielh Neelsen modificado para Cryptosporidium spp., según las técnicas establecidas (21).

Las muestras para coprocultivo se sembraron en agar sangre, MacConkey, Salmonella Shigella (SS), Hecktoen, xilosa-lactosa-desoxicolato (XLD), MacConkey sorbitol, tiosulfato-citrato-bilissacarosa (TCBS), de Difco® y cefsulodin-irgasannovobiocin (CIN, medio selectivo para Yersinia spp.) de Oxoid@; éstas se enviaron al laboratorio de investigación en infectología pediátrica de la Universidad de Antioquia en Medellín. A las colonias sospechosas de enteropatógenos se les realizó oxidasa (Difco®) y serie bioquímica tradicional (TSI, LIA, citrato, SIM y urea, de Difcoß); dependiendo del resultado de estas pruebas, se hizo la identificación mediante aglutinación en placa con los antisueros respectivos $(B B L \circledast)$ y prueba de sensibilidad por el método de Kirby Bauer.

\section{Análisis estadístico}

Para el análisis de los datos se usaron los paquetes estadísticos StatXact 4.0 y Statistica 98. Se compararon los valores de las variables medidas tanto al ingreso del estudio, como al final del período de hidratación y del período de observación, para los dos grupos de niños, usando la prueba $t$ de Student de muestras independientes. Las comparaciones en cada grupo se hicieron usando la prueba t pareada.

Las comparaciones de frecuencias en los dos grupos se llevaron a cabo mediante la prueba exacta de Freeman-Halton; esta prueba admite frecuencias pequeñas, inclusive cero. Se evaluó el riesgo relativo de la necesidad de utilizar líquidos intravenosos. Las comparaciones y pruebas se consideraron significativas cuando $p<0,05$.

\section{Resultados}

Cincuenta y cuatro niños recibieron el suero SRO/ OMS (grupo 1) y 47 la solución a base de harina de plátano (SRO/plátano) (grupo 2). No se encontraron diferencias estadísticamente significativas en las características del ingreso entre ambos grupos (cuadro 1).

Tampoco se presentó diferencia estadísticamente significativa entre los grupos en relación con el estado nutricional (cuadro 2).

No se presentaron diferencias estadísticamente significativas en relación con los agentes productores de diarrea identificados en ambos grupos (cuadro 3).

\section{Período de hidratación}

Los datos referentes al período de hidratación se presentan en el cuadro 4 . La hidratación tuvo éxito en 51 niños $(94,4 \%)$ del grupo SRO/OMS y en 43 $(91,5 \%)$ del grupo $S R O / p l a ́ t a n o(p=0,70)$. Los tres

Cuadro 1. Características al ingreso de los niños incluidos en cada uno de los dos grupos de estudio.

\begin{tabular}{llllll}
\hline & \multicolumn{2}{l}{ Grupo SRO/OMS (n=54) } & \multicolumn{2}{l}{ Grupo SRO/plátano (n=47) } \\
& Media & DE & Media & DE & p \\
\hline Edad (meses) & 12,1 & 9,0 & 12,0 & 7,55 & 0,93 \\
Diarrea (días) & 3,4 & 2,4 & 4,0 & 2,9 & 0,25 \\
Deposiciones al día & 8,8 & 4,0 & 9,2 & 4,4 & 0,56 \\
Sangre en las heces (n) (\%) & $2(3,7)$ & 19,1 & $2(4,2)$ & 20,4 & 0,64 \\
Vómitos por día & 4,4 & 3,2 & 4,4 & 3,3 & 0,90 \\
Antibióticos (n) (\%) & $14(25,9)$ & 44,2 & $15(31,9)$ & 47,1 & 0,52 \\
Antiparasitarios(n) (\%) & $6(11,1)$ & 31,7 & $4(8,51)$ & 28,2 & 0,75 \\
Antidiarreicos (n) (\%) & $1(1,8)$ & 13,6 & $0(0,0)$ & 0,0 & 0,41 \\
Peso al ingreso (g) & 7.924 & 2.118 & 8.263 & 2.038 & 0,72 \\
Talla (cm) & 72,0 & 9,0 & 72,6 & 9,1 & 0,31 \\
Leche materna (n) (\%) & $18(33,3)$ & 47,6 & $21(44,7)$ & 50,3 & \\
\hline
\end{tabular}


Cuadro 2. Estado nutricional peso para la talla en unidades $Z$ de los niños incluidos en cada uno de los grupos de estudio.

\begin{tabular}{lrrrr}
\hline & Grupo SRO/OMS ( $\mathbf{n = 5 4 )}$ & \multicolumn{2}{c}{ Grupo SRO/plátano (n=47) } \\
\hline Exceso de peso (>DE) & 2 & $3,7 \%$ & 2 & $4,2 \%$ \\
Normal (entre -1 y +1 DE) & 18 & $33,3 \%$ & 22 & $46,8 \%$ \\
Desnutrición leve (entre -1 y -2 DE) & 27 & $50,0 \%$ & 13 & $27,7 \%$ \\
Desnutrición moderada (entre -2 y -3 DE) & 7 & $13,0 \%$ & 10 & $21,3 \%$ \\
$\mathrm{p}=0,14$ & & & & \\
\hline
\end{tabular}

niños que no se pudieron hidratar en el grupo SRO/ OMS y los cuatro que no se hidrataron en el grupo $\mathrm{SRO} /$ plátano se consideraron fracasos tempranos, terminaron de hidratarse con solución polielectrolítica por vía intravenosa y se excluyeron del estudio a partir de ese momento.

No se presentaron diferencias estadísticamente significativas en relación con el tiempo de hidratación.

El gasto fecal durante el periodo de hidratación se analizó en todos los pacientes, incluso los éxitos y los fracasos, hasta el momento en que fue necesario administrar líquidos intravenosos. El promedio del gasto fecal durante el período de hidratación en el grupo SRO/OMS fue de $7,5 \mathrm{~g} / \mathrm{kg}$ por hora $(D E= \pm 7,6)$ y de $7,0 \mathrm{~g} / \mathrm{kg}$ por hora $(D E= \pm 9,4)$ para el grupo $S R O /$ plátano $(p=0,78)$.

El gasto fecal de los niños que presentaron fracaso temprano fue de $3,3 \mathrm{~g} / \mathrm{kg}$ por hora $(\mathrm{DE}= \pm 2,9)$ en el grupo SRO/OMS y de $16,7 \mathrm{~g} / \mathrm{kg}$ por hora $(\mathrm{DE}= \pm 19,2)$ en los que fracasaron en el grupo SRO/ plátano $(p=0,29)$. De los tres fracasos en el grupo $\mathrm{SRO} / \mathrm{OMS}$, dos fueron por vómito persistente, uno se retiró a las dos horas y otro a las cinco; durante este período no habían presentado deposición. El tercer niño tuvo diarrea de gasto alto. Los cuatro niños en los que se consideró fracaso en el grupo $\mathrm{SRO} /$ plátano tuvieron gasto fecal alto, en especial uno de ellos que se retiró a la quinta hora cuando llevaba un gasto fecal de 63,9 g/kg por hora. Este fue un caso de diarrea muy abundante, fuera de lo común, en el cual se encontró Giardia lambia.

Si se excluyen los siete niños en los que hubo fracaso en los dos grupos, el gasto fecal en el grupo SRO/OMS fue de $7,7 \mathrm{~g} / \mathrm{kg}$ por hora $(\mathrm{DE}= \pm 7,7)$ y en el grupo SRO/plátano, $6,1 \mathrm{~g} / \mathrm{kg}$ por hora $(\mathrm{DE}= \pm 7,8)(p=0,31)$.

El volumen del suero necesario para conseguir la

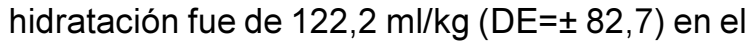

Cuadro 3. Prevalencia de infección por diferentes microorganismos en los niños de los dos grupos de estudio.

\begin{tabular}{|c|c|c|c|c|c|}
\hline & \multicolumn{2}{|c|}{ Grupo SRO/OMS } & \multicolumn{2}{|c|}{ Grupo SRO/plátano } & \multirow[t]{2}{*}{$\mathbf{p}$} \\
\hline & $\#$ & $\%$ & $\#$ & $\%$ & \\
\hline Látex para rotavirus & $n=39$ & & $n=3$ & & \\
\hline Rotavirus positivo & 12 & 30,8 & 5 & 3,2 & 0,10 \\
\hline Coprocultivo & $n=39$ & & $\mathrm{n}=3$ & & \\
\hline Salmonella spp. & 3 & 7,7 & 4 & 11,8 & 0,70 \\
\hline Coprológico & $n=46$ & & $n=4$ & & \\
\hline Ascaris lumbricoides & 9 & 19,6 & 3 & 7,5 & 0,13 \\
\hline Trichuris trichiura & 6 & 13,0 & 3 & 7,5 & 0,49 \\
\hline Uncinaria sp. & 1 & 2,2 & 0 & 0,0 & 0,53 \\
\hline Strongyloides stercolaris & 1 & 2,2 & 0 & 0,0 & 0,53 \\
\hline Giardia lamblia & 2 & 4,3 & 3 & 7,5 & 0,66 \\
\hline Entamoeba histolytica & 1 & 2,5 & 1 & 2,5 & 0,66 \\
\hline Balantidium coli & 0 & 0,0 & 1 & 2,5 & 0,26 \\
\hline Tinción para Cryptosporidium & $n=44$ & & $n=3$ & & \\
\hline Cryptosporidium parvum & 1 & 2,2 & 0 & 0 & 0,12 \\
\hline Tinción para Campylobacter & $n=40$ & & $n=3$ & & \\
\hline Campylobacter jejuni & 0 & 0,0 & 1 & 3,0 & 0,45 \\
\hline
\end{tabular}


grupo SRO/OMS y de $111,1 \mathrm{ml} / \mathrm{kg}(\mathrm{DE}= \pm 91,2)$

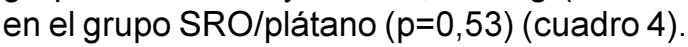

La ganancia de peso durante el período de hidratación fue de $4,1 \%$ en el grupo SRO/OMS y de

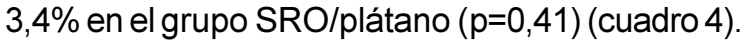

Los niveles plasmáticos de sodio al ingreso fueron menores en el grupo SRO/OMS $(p=0,01)$. Al final del período de hidratación no se presentaron diferencias estadísticamente significativas en las concentraciones de sodio entre los grupos $(p=0,30)$. Dos niños desarrollaron hiponatremia en el grupo SRO/OMS y tres en el grupo SRO/plátano. Ninguno presentó síntomas (cuadro 5).

No se presentaron diferencias estadísticamente significativas en los niveles séricos de potasio, ni al principio ni al final de la hidratación, en los dos grupos (cuadro 5).

\section{Período de observación}

Se consideró como período de observación el tiempo durante el cual los niños recibieron suero oral; para los casos exitosos fue de veinticuatro horas, para los fracasos tempranos y tardíos fue el tiempo durante el cual recibieron suero oral. El promedio del período de observación, incluidos éxitos y fracasos, fue de 22,3 horas $(D E= \pm 5,3$ ) en el grupo SRO/OMS y de 22,7 horas (DE $= \pm$ $4,7)$ en el grupo SRO/plátano ( $p=0,73$ ) (cuadro 6).

Hubo 3 niños en el grupo SRO/OMS que, después de haberse hidratado por vía oral, se deshidrataron de nuevo y fue necesario administrarles líquidos intravenosos antes de completar las primeras veinticuatro horas a partir de su ingreso. Estos niños se consideraron fracasos tardíos y también fueron excluidos del estudio a partir de ese momento. En el grupo SRO/plátano, ninguno de los niños se volvió a deshidratar durante el período de observación.

Los niños que en algún momento necesitaron líquidos intravenosos fueron $6(11,1 \%)$ en el grupo SRO/OMS y $4(8,5 \%)$ en el grupo SRO/plátano (riesgo relativo $=1,31$; IC 95\%: 0,39 a 4,35).

Como el periodo de observación no fue igual para todos los niños, los resultados no se presentan en términos absolutos, sino considerando el número de horas durante las cuales cada niño permaneció en el estudio.

Cuadro 4. Características del proceso de hidratación en los dos grupos de estudio.

\begin{tabular}{|c|c|c|c|c|c|}
\hline \multirow[b]{2}{*}{ Éxitos } & \multicolumn{2}{|c|}{ Grupo SRO/OMS } & \multicolumn{2}{|c|}{ Grupo SRO/plátano } & \multirow{2}{*}{$\frac{\mathbf{p}}{0,70}$} \\
\hline & $\begin{array}{l}51 / 54 \\
\text { Media }\end{array}$ & $\begin{array}{l}94,4 \% \\
D E\end{array}$ & $\begin{array}{l}43 / 47 \\
\text { Media }\end{array}$ & $\begin{array}{l}91,5 \% \\
\text { DE }\end{array}$ & \\
\hline Horas de hidratación & 5,8 & 3,0 & 6,2 & 3,7 & 0,51 \\
\hline Suero necesario para hidratarse $(\mathrm{ml} / \mathrm{kg})$ & 122,2 & 82,7 & 111,1 & 91,2 & 0,53 \\
\hline Suero necesario para hidratarse ( $\mathrm{ml} / \mathrm{kg}$ por hora) & 19,5 & 6,9 & 20,2 & 6,4 & 0,50 \\
\hline Gasto fecal $\mathrm{g} / \mathrm{kg}$ por hora * & 7,5 & 7,6 & 7,0 & 9,4 & 0,78 \\
\hline Peso al ingreso $(\mathrm{g})$ & 7.924 & 2.118 & 8.263 & 2.038 & 0,41 \\
\hline Peso al final del periodo de hidratación (g) & 8.283 & 2.153 & 8.746 & 1.874 & 0,27 \\
\hline Ganancia de peso $(\mathrm{g})$ & 303,4 & 280,3 & 268,6 & 270,6 & 0,54 \\
\hline Porcentaje de peso ganado & 4,1 & 4,5 & 3,4 & 3,3 & 0,41 \\
\hline
\end{tabular}

* El gasto fecal durante el período de hidratación incluye todos los pacientes.

Cuadro 5. Concentraciones de electrolitos al inicio y al final del período de hidratación en los dos grupos de estudio.

\begin{tabular}{lccccccc}
\hline & \multicolumn{3}{c}{ Grupo SRO/OMS } & & \multicolumn{2}{c}{ Grupo SRO/plátano } \\
& $\mathbf{n}$ & Media & DE & n & Media & DE & p \\
\hline Sodio inicial & 45 & 136,2 & 5,8 & 43 & 140,3 & 8,8 & 0,01 \\
Sodio al final del periodo de hidratación & 42 & 136,5 & 5,8 & 42 & 138,2 & 8,44 & 0,30 \\
Potasio inicial & 45 & 4,30 & 0,80 & 43 & 4,08 & 0,99 & 0,24 \\
Potasio al final del periodo de hidratación & 41 & 4,35 & 0,83 & 41 & 4,10 & 0,96 & 0,37 \\
\hline
\end{tabular}


El gasto fecal durante el período de observación fue $5,7 \mathrm{~g} / \mathrm{kg}$ por hora ( $D E= \pm 4,7$ ) en el grupo SRO/ OMS y de $6,3 \mathrm{~g} / \mathrm{kg}$ por hora en el grupo SRO/ plátano $(\mathrm{DE}= \pm 7,9)(\mathrm{p}=0,73)$ (cuadro 6).

Si se excluyen los fracasos, el gasto fecal durante el período de observación en el grupo SRO/OMS fue de $5,5 \mathrm{~g} / \mathrm{kg}$ por hora (DE= $\pm 4,4$ ) y de $5,3 \mathrm{~g} / \mathrm{kg}$ por hora $(D E:= \pm 5,5)$ en el grupo $\mathrm{SRO} /$ plátano $(p=0,85)$.

No se presentaron diferencias estadísticamente significativas en relación con la ingestión de suero, el consumo de fórmula y de sopa, la eliminación urinaria, el volumen y número de los vómitos, durante el período de observación. Tampoco se presentaron diferencias en relación con la cantidad de niños que recibieron leche materna durante este período (cuadro 6).

\section{Discusión}

La solución de hidratación oral a base de harina de plátano precocida con electrolitos estandarizados demostró ser segura y efectiva para corregir la deshidratación. Sin embargo, en este estudio no se pudo comprobar su efecto antidiarreico.

La hipótesis sobre el efecto antidiarreico del plátano tiene origen popular y es muy frecuente en algunas regiones la preparación en el hogar de bebidas o sopas con las diversas variedades de plátano verde. El estudio anterior realizado por nuestro grupo (9) utilizó la receta casera ya descrita, con la variedad de plátano hartón $(M$. paradisiaca); la osmolaridad de esta solución en catorce muestras fue de $134,4 \mathrm{mOsm} / \mathrm{L}$ $(\mathrm{DE}= \pm 12,3)$. En esa investigación se encontró que los niños que recibieron la bebida de plátano tuvieron un gasto fecal durante el período de hidratación de $4,69 \mathrm{~g} / \mathrm{kg}$ por hora $(\mathrm{DE}= \pm 4,9)$ mientras el gasto fecal en los que recibieron la solución estándar fue de $8,45 \mathrm{~g} / \mathrm{kg}$ por hora $(\mathrm{DE}= \pm$ $9,7)$; esta diferencia fue significativa $(p=0,0001)$.

En el presente estudio no se encontró disminución significativa del gasto fecal entre los niños que recibieron la solución a base de harina de plátano precocida con electrolitos estandarizados, ni durante el período de hidratación, ni durante las veinticuatro horas posteriores a la hospitalización.

Algunas de las explicaciones para la diferencia en el gasto fecal durante el período de hidratación entre los niños que recibieron las solucionesa base de plátano en los dos estudios, son: en el estudio anterior se utilizó una solución preparada en forma casera, con $50 \mathrm{~g}$ de harina de plátano y $3,5 \mathrm{~g}$ de sal; esa solución tenía una osmolaridad de 134 $\mathrm{mOsm} / \mathrm{L}(\mathrm{DE}= \pm 12,3)$; en el presente estudio se trabajó con harina de plátano precocida con los electrolitos estandarizados en iguales concentraciones que en el suero estándar, con una osmolaridad de la solución de $226 \mathrm{mOsm} / \mathrm{L}$

Cuadro 6. Parámetros de los dos grupos de estudio durante el período de observación

\begin{tabular}{llllll}
\hline & \multicolumn{2}{l}{ Grupo SRO/OMS } & \multicolumn{2}{c}{ Grupo SRO/plátano } \\
& Media & DE & Media & DE & p \\
\hline Tiempo de observación (horas) & 22,3 & 5,3 & 22,7 & 4,7 & 0,73 \\
Gasto fecal g/kg por hora & 5,7 & 4,7 & 6,3 & 7,9 & 0,67 \\
Número de deposiciones & 8,2 & 4,8 & 8,4 & 5,8 & 0,86 \\
Suero ingerido (ml/kg por hora) & 12,8 & 9,7 & 10,1 & 6,5 & 0,10 \\
Volumen de fórmula (ml/g por /hora) & 0,70 & 0,92 & 0,58 & 1,17 & 0,56 \\
Recibieron leche materna (n) (\%) & $16(29,6)$ & 6,2 & $20(42,6)$ & 7,2 & 0,18 \\
Número de tomas de leche materna & 6,6 & 3,6 & 6,9 & 3,7 & 0,78 \\
Eliminación urinaria (ml/kg por hora) & 0,69 & 0,68 & 0,66 & 0,58 & 0,85 \\
Volumen de vómito (g/kg por hora) & 0,24 & 0,65 & 0,28 & 0,49 & 0,77 \\
Número promedio de vómitos* & 2,5 & 1,9 & 2,9 & 1,9 & 0,48 \\
Peso al final de la observación (g) & 8.267 & 2.174 & 8.505 & 2.057 & 0,57 \\
Ganancia de peso (g) & 343 & 288 & 242 & 293 & 0,08 \\
Porcentaje de peso ganado al final & 4,6 & 4,2 & 3,1 & 3,4 & 0,53 \\
\hline
\end{tabular}

* Entre los pacientes que tuvieron vómitos: 27 en el grupo 1 y 26 en el grupo 2. 
$(\mathrm{DE}= \pm 9,16)$. Es posible que la menor osmolaridad de la mezcla utilizada en el primer estudio esté relacionada con el menor gasto fecal. Los estudios que han utilizado agua de arroz sin electrolitos cuya osmolaridad es muy baja, han mostrado menor gasto fecal en relación con el suero estándar $(22,23)$; estas soluciones son útiles para prevenir la deshidratación en el hogar; sin embargo, no se recomiendan para tratar la deshidratación, debido al riesgo de hiponatremia.

En el primer estudio con suero preparado en forma casera con harina de plátano la concentración de sodio fue se $60,52 \mathrm{mmol} / \mathrm{L}(\mathrm{DE}= \pm 8,87$ ). En ese estudio se encontró disminución de los niveles plasmáticos de sodio en forma significativa, y algunos niños desarrollaron hiponatremia asintomática. En la solución utilizada en el presente estudio, las concentraciones de electrolitos estaban estandarizadas como en el suero de referencia y la concentración de sodio fue de $90 \mathrm{mmol} / \mathrm{L}$. En la búsqueda de la fórmula más adecuada para una solución de hidratación oral de baja osmolaridad, también se encontró que los niños que recibían las soluciones con $60 \mathrm{mmol} /$ $\mathrm{L}$ de sodio y osmolaridad total de $224 \mathrm{mOsm} / \mathrm{L}$, tenían gasto fecal significativamente menor. En cambio, cuando el contenido de sodio era de 75 $\mathrm{mmol} / \mathrm{L}$ y la osmolaridad de $245 \mathrm{mOsm} / \mathrm{L}$, el gasto fecal no era significativamente menor que en los que recibían suero estándar. El problema de los sueros con $60 \mathrm{mmol} / \mathrm{L}$ de sodio es que originan un mayor riesgo de hiponatremia (11). La reducción del gasto fecal en el primer estudio pudo estar condicionada a la menor concentración de sodio en la solución.

Otra posible explicación puede ser la técnica de preparación de la harina de plátano. Aunque en ambos estudios se utilizó el plátano hartón $(M$. paradisiaca), en el primer estudio se extrajo la harina del plátano crudo, en tanto que en el presente estudio se utilizó harina de plátano previa cocción en agua. Quizá la técnica de preparación de la harina juegue un papel importante en la disminución del gasto fecal como ocurrió en el primer estudio.

Al analizar los niveles séricos de potasio entre la solución casera del estudio anterior y la solución actual utilizada en el presente estudio se encuentra que la solución casera no tenía adición de potasio; sin embargo, el plátano le daba una concentración de potasio a la solución de $13 \mathrm{mmol} /$ L. En el primer estudio se encontró disminución de los niveles plasmáticos de potasio entre los niños que recibieron la solución a base de plátano, aun cuando no se presentaron manifestaciones clínicas de hipopotasemia. En el presente estudio, en el cual el suero contenía $20 \mathrm{mmol} / \mathrm{L}$ de potasio, además del potasio que pudiera estar aportando el plátano, no se presentaron diferencias en la concentración de potasio al final de la hidratación entre los dos grupos.

En este estudio, la necesidad de utilizar líquidos intravenosos fue baja en los dos grupos: $11,1 \%$ entre los niños que recibieron el suero estándar y $8,5 \%$ entre quienes recibieron la solución a base de harina de plátano, diferencia que no fue significativa. Los fracasos que se presentaron entre los niños que recibieron SRO/plátano fueron tempranos y se debieron a gasto fecal elevado; en este grupo no se presentaron fracasos tardíos. Los fracasos ocurridos entre los niños que recibieron SRO/OMS fueron tanto tempranos como tardíos y, predominantemente, por vómito persistente. En el metaanálisis de los sueros de baja osmolaridad se encontró que la necesidad de utilizar líquidos intravenosos entre los niños que recibian la solución estándar con $90 \mathrm{mmol} / \mathrm{L}$ de sodio y osmolaridad de $311 \mathrm{mOsm} / \mathrm{L}$ era de $15 \%$ y en los que recibían la solución de baja osmolaridad con concentraciones de sodio entre 60 y $75 \mathrm{mmol} / \mathrm{L}$ y osmolaridad entre 224 y 245 $\mathrm{mOsm} / \mathrm{L}$, era de $10 \%$. Esta diferencia fue significativa, teniendo en cuenta el número elevado de niños incluidos en el metaanálisis (16).

Los dos grupos fueron comparables al ingreso, excepto en la concentración plasmática de sodio que fue significativamente menor en el grupo que recibió SRO/OMS $(p=0,01)$, diferencia que se atribuye al azar.

El plátano en sus distintas variedades es utilizado frecuentemente por la comunidad para preparar líquidos y alimentos para administrar a los niños durante las enfermedades diarreicas (24). Los líquidos caseros preparados con harina de plátano 
son efectivos para prevenir la deshidratación y tienen la ventaja de que aportan potasio. El plátano es la base de la alimentación de algunas comunidades en Colombia y otros países. En la alimentación de los niños se utiliza como base de la preparación de sopas y "coladas". De acuerdo con su disponibilidad, el plátano es útil e importante siempre y cuando haga parte de una dieta que suministre otros alimentos fuentes de proteínas, grasas y micronutrientes. De hecho, en la Unidad Vida Infantil se está utilizando el plátano para preparar mezclas para la recuperación de niños con desnutrición grave. Estas mezclas son a base de leche, agua, plátano, aceite vegetal y oligoelementos.

Después de más de veinticinco años de venir recomendando una sola fórmula para la hidratación oral, la OMS aceptó recientemente cambiar oficialmente la fórmula del suero oral por uno de baja osmolaridad. Este nuevo suero contiene las siguientes concentraciones de electrolitos, en $\mathrm{mmol} / \mathrm{L}$ : sodio, 75 ; potasio, 20 ; cloruro, 65 ; citrato, 10 , y glucosa, 75 . Su osmolaridad es de 245 $\mathrm{mOsm} / \mathrm{L}$ La ventaja más importante de esta nueva fórmula es la disminución de la proporción de niños que necesitan líquidos intravenosos durante las primeras veinticuatro horas de iniciada la hidratación oral. Algunos estudios demostraron disminución significativa del gasto fecal y del tiempo de duración de la enfermedad. La nueva fórmula tiene ventajas, pero todavía no llena las expectativas del "supersuero".

Es muy importante continuar con la búsqueda de una solución que disminuya el riesgo de muerte por deshidratación y disminuya el gasto fecal, con un producto disponible en la región, que tenga un efecto positivo sobre la nutrición de los niños, que haga parte del acervo cultural alimentario o etnomédico de los grupos, de tal manera que pueda estar disponible y ser apropiado por la colectividad.

\section{Agradecimientos}

A Colciencias (código: 1115-04-10219) y Augura que financiaron la mayor parte de los costos directos de la investigación. Al Hospital Universitario San Vicente de Paúl de Medellín que autorizó la realización sin cobro de los ionogramas en el laboratorio de nefrología pediátrica. A Corpaúl que financió la preparación de sobres de suero oral sin glucosa, para la preparación del suero basado en harina de plátano.

A las directivas y al personal administrativo, médico y paramédico del Hospital Francisco Valderrama de Turbo que colaboraron para la realización del estudio.

A los siguientes residentes de Pediatría que rotaron cada uno un mes por la Unidad Vida Infantil y participaron en la atención de los pacientes: Nelson Gómez, Víctor Hugo Giraldo, Olga Lucía Morales, John Edgar Lopera, María Beatriz Ruiz, Javier Enrique Fox, Milton Jiménez, Sandra Silva y Olga Elena Aguirre.

A los siguientes internos que rotaron durante tres meses por la Unidad en calidad de jóvenes investigadores y también estuvieron a cargo de la atención de los niños: Iván Darío Flórez, Juan Camilo Jaramillo, Natalí Castaño, Natalia Figueroa, Paula Flórez, Liliana Wilches, Juan Pablo Bedoya, Gladys Nadieska Sánchez, Alejandra Sañudo, Juan José Yepes, Alejandro Marín, Alexandra Salazar, Sujey Gómez, María Victoria Lopera y Maribel Mahecha.

A las siguientes auxiliares de enfermería que estuvieron a cargo de la atención de los pacientes y de los registros: Maritza Lemos, Marisa Osorio, Sandra Mosquera, Rosalba Acevedo y Josefina López. A los bacteriólogos Carmen Tulia Zapata Muñoz, Harold Eduardo Durango y Libertad Aguilar y al estudiante de bacteriología Carlos Alberto Rivero, que procesaron los exámenes de laboratorio.

\section{Conflicto de intereses}

Los autores manifiestan que no existe ningún conflicto de interés.

\section{Financiación}

El estudio fue financiado por Colciencias, la Asociación de Bananeros de Colombia, Augura y la Universidad de Antioquia.

\section{Referencias}

1. Fondo de las Naciones Unidas para la Infancia. Estado mundial de la infancia. Ginebra: UNICEF; 1998. p.131. 
2. Arias MM, Bernal C, Pérez L, Lalinde MI, Ricaurte CF, Vargas M. Características del proceso enfermedad-atención-muerte en niños con diarrea. Antioquia, Colombia. Bol Med Hosp 2001;58:320-9.

3. The International Study Group on Improved ORS. Impact of glycine-containing ORS solutions on stool output and duration of diarrhoea: a metaanalysis of seven clinical trials. Bull WHO 1991;69:541-8.

4. Gore SM, Fontaine O, Pierce NF. Impact of rice based oral rehydration solution on stool output and duration of diarrhoea: metaanalysis of 13 clinical trials. BMJ 1992; 304:287-91

5. Kenya PR, Odongo HW, Oundo G. Cereal based oral rehydration solutions. Arch Dis Child 1989;64:1032-5.

6. Santos Ocampo PD, Bravo IC, Rogacion JM, Battad GR. A randomized double-blind clinical trial of a maltodextrin-containing oral rehydration solution in acute infantile diarrhea. J Pediatr Gastroenterol Nutr 1993;16: 23-8.

7. Sack RB, Castrellon J, Della Sera E. Hydrolyzed lactalbumin-based oral rehydration solution for acute diarrhoea in infants. Acta Paediatr 1994;83:819-24.

8. Lebenthal E, Khin-maung-U, Rolson DDK, KhinMyat-Tun, Tin-Nu-Swe, Tthein-Thein-Myint et al. Termophilic amylase-digested rice-electrolyte solution in the treatment of acute diarrhea in children. Pediatrics 1995;95:198-202.

9. Arias MM, Alcaraz G, Bernal C, González G. Oral rehydration with a plantain-flour based solution in children dehydrated by acute diarrhea: a clinical trial. Acta Paediatr 1997;86:1047-51.

10. El-Mougi M, El-Akkad N, Hendaw A, Hassan M, Amer A, Fontaine $O$ et al. Is a low-osmolarity ORS solution more efficacious than standard WHO ORS solution? J Ped Gastroenterol Nutr 1994;19:83-6.

11. International Study Group on Reduced Osmolarity ORS Solutions. Multicentre evaluation of reduced-osmolarity oral rehydration salts solution. Lancet $1995 ; 345: 282-5$.

12. Santosham M, Fayad I, Ziki M, Hussein A, Amponsah A, Duggan C. A double-blind clinical trial comparing the World Health Organization oral rehydration solution with a reduced osmolarity solution containing equal amounts of sodium and glucose. J Ped 1996;128:45-51.
13. Bernal C, Velásquez C, García G, Palacio C, Uribe G. Hidratación oral con una solución de baja osmolaridad. Biomédica 2003;23:47-59.

14. CHOICE Study Group. Multicenter randomized double blind clinical trial to evaluate the efficacy and safety of a reduced osmolarity oral rehydration salts solution in children with acute watery diarrhea. Pediatrics 2000;107: 613-8.

15. Alcaraz G. Solución de rehidratación oral preparada a base de harina de plátano. Estudio bioquímico. Invest Educ Enferm 1994;12:38-9.

16. Hahn SK, Kim YJ, Garner P. Reduced osmolarity oral rehydration solution for treating dehydration due to diarrhoea in children: systematic review. BMJ 2001;323: $81-5$.

17. World Heath Organization. Reduced osmolarity oral rehydration salts (ORS) formulation. Report from a meeting of experts jointly organized by UNICEF and WHO. New York, USA, July 18, 2001. WHO/FCH/CAH/ 01.22. Geneva: WHO; 2001.

18. Pocock SJ. Clinical trials. A practical approach. Chichester: John Wiley \& sons; 1987. p.266.

19. OPS/OMS. Manejo del paciente con diarrea. Programa de control de enfermedades diarreicas. Tercera edición. Washington, D.C.: OPS; 1991.

20. Hamil PVV, Drizid TA, Johnson CL, Reed RB, Roche AF, Moore WM. Physical growth: National Center for Health Statistics percentiles. Am J Clin Nutr 1979;32:60729.

21. World Health Organization. Programme of control of diarrhoeal diseases. Manual for laboratory investigations of acute enteric infections. CDD/83.3 Rev 1. Geneva: WHO; 1987

22. Metha MN, Subramaiam S. Comparison of rice water, rice electrolyte solution, and glucose electrolyte solution in the management of infantile diarrhoea. Lancet $1986 ; 1: 843-5$.

23. Martínez-Salgado H, Calva-Mercado JJ, MotaHernández F, Posadas-Tello L, Bross-Soriano D. Eficacia de una bebida a base de arroz en el manejo de la deshidratación por diarrea aguda en niños. Bol Med Hosp Infant Mex 1991;48:544-52.

24. Bernal C, Cañarte D, Gutiérrez EL. Líquidos disponibles en el hogar para prevenir la deshidratación. Bol Med Hosp Infant Mex 1994;51:7-14. 